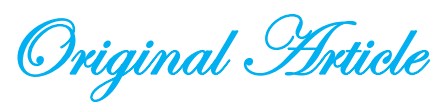

\title{
Reliability of agglutination test to detect alloantibodies in patients with repeated blood transfusion
}

\begin{abstract}
Background: Blood transfusion is an integral part in the management of sickle cell disease patients. Allogeneic blood transfusion is a form of temporary transplantation. A recipient often mounts an immune response to the donor antigens resulting in various clinical consequences including delayed hemolytic transfusion reactions. Delayed reaction is often seen in individuals who have received repeated transfusion of ABO compatible blood that incompatible for other blood group antigens because of minor allelic difference stimulate the production of IgG antibodies. In the patients who have sickle cell disease the majority of tests may have low sensitivity and in turn may fail to show the autoantibodies.

Objectives: This study has been conducted for detection of allo-antibodies in patient with sickle cell anaemia and hemophilia who received repeated blood transfusions using newly introduced test system; the DiaMed-Immuno-Diffusion microtyping system.

Methods: Samples were collected randomly from 60 patients with repeated blood transfusions. Micro column gel test as well as agglutination method were performed for all samples. All the results were analyzed using Statistical Packages of Social Sciences (SPSS).

Results and Discussion: This test provides clear and stable reactions that improve result interpretation. It proved to be more sensitive than the conventional tube agglutination technique as it captures agglutinate in a semi solid medium and on the other hand it has the capacity to detect unexpected antibodies. This in turn enhances visibility of agglutination compared to the traditional Tube techniques.
\end{abstract}

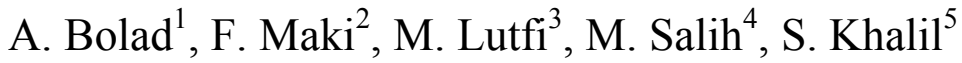

Keywords: Allogeneic, allo-antibodies, sickle cell disease, DiaMed-ID system.

$\mathrm{T}$ The major barrier to successful blood transfusion is the immune responses to the cell surface molecules that differ between individuals; the most important alloantigen system in blood transfusion is the ABO system ${ }^{1,2}$. One of the main complications of red blood cells (RBCs) transfusion is the development of alloimmunization to RBCs antigens ${ }^{3,4}$. Among serious complication of alloimmunization is delayed hemolytic transfusion reaction/hyperhemolysis (DHTR/H) syndrome ${ }^{5,6}$.

The transfused blood induces clonal selection

1. Alneelain University, Faculty of Medicine, Department of Microbiology and Unit of Immunology, Alneelain Medical Research Centrer.

2. Jaffar Ibn Oaf Pediatric Hospital, Blood Bank

3, Alneelain University, Faculty of Medicine

4. Ahfad University, Faculty of Laboratory Sciences

Correspondence: E.mail:aaabolad@hotmail.com and production of $\mathrm{IgG}$ against common membrane antigens e.g. Rh Kidd, kell and Duffy blood groups. IgG is less effective than IgM in activating complement so the lysis is incomplete and many of the transfused cells are destroyed at extravascular sites by agglutination opsonization and sub sequent phagocytes by macrophages. This has mostly been described in sickle cell anemia ${ }^{7-10}$ but occasionally in beta-thalassemia ${ }^{11}$. The antibodies to minor antigen (unexpected antibodies) are capable of causing hemolytic transfusion reactions ${ }^{12}$.On the other hand, antibodies may triggers an immediate hemolytic reaction, resulting in both intravascular lysis of red blood cells probably mediated by the complement system, and extensive phagocytes of antibody- and complement- coated erythrocytes by macrophages of the liver and spleen ${ }^{13}$. 
However, in the patients who have sickle cell disease the majority of tests may have low sensitivity and in turn may fail to show the autoantibodies $^{14,15}$. In the $1990 \mathrm{~s}$, the micro column gel technique was introduced for screening and identification of such unexpected antibodies ${ }^{16}$. This method is not only easy to perform but, also easy to standardize and read, so it has become the most common technique in the blood bank laboratories of many countries.

Objectives: This study aimed to assess the ability of agglutination test to detect alloantibodies using conditional ratios (sensitivity, specifity, positive and negative predictive values and accuracy). Probably, this is the first study in Sudan in this field.

\section{Patients and Methods:}

Ethical consideration The study had received an ethical approval from the ethical review committee at the Institute of Tropical Medicine University of Sudan academy. The samples were collected (the donated units) by well trained medical laboratory technicians at the blood banks.

Study population The study was conducted in 60 patients with repeated blood transfusions. Patients were recruited from Khartoum Teaching Hospital and Children Emergency Hospital. Serum samples were considered for analysis following obtaining formal consent from blood donors then, has been cryopreserved until using at $-20^{\circ} \mathrm{C}$.

Diamed - id micro typing system and agglutination method: Micro column gel test was done as essentially prescribed by Lapierre et al., $1990^{16}$. Agglutination method was also performed for all samples using tube agglutination technique.

Data Analysis: Statistical analysis was performed with statistical software SPSS for Windows (version 17.0). Crosstabulation was performed for the findings of agglutination and immunodiffusion tests. The conditional ratios (sensitivity, specifity, positive and negative predictive values and accuracy) were calculated for agglutination test accordingly.

\section{Results}

The study involved 60 patients with repeated blood transfusions. Fifty patients (83.4\%) were suffering from sickle cell anemia. The rest were suffering from hemophilia (10\%) thrombocytopenia $(3.3 \%)$ and other types of anemias (3.3\%). Forty patients (67.7\%) underwent five or more blood transfusions. Nine patients $(15 \%)$ of studied patients were having alloantntibodies according to the immunodiffusion test. Only $22.2 \%$ of positive immunodiffusion test, were also positive when using agglutination test (figure 1).

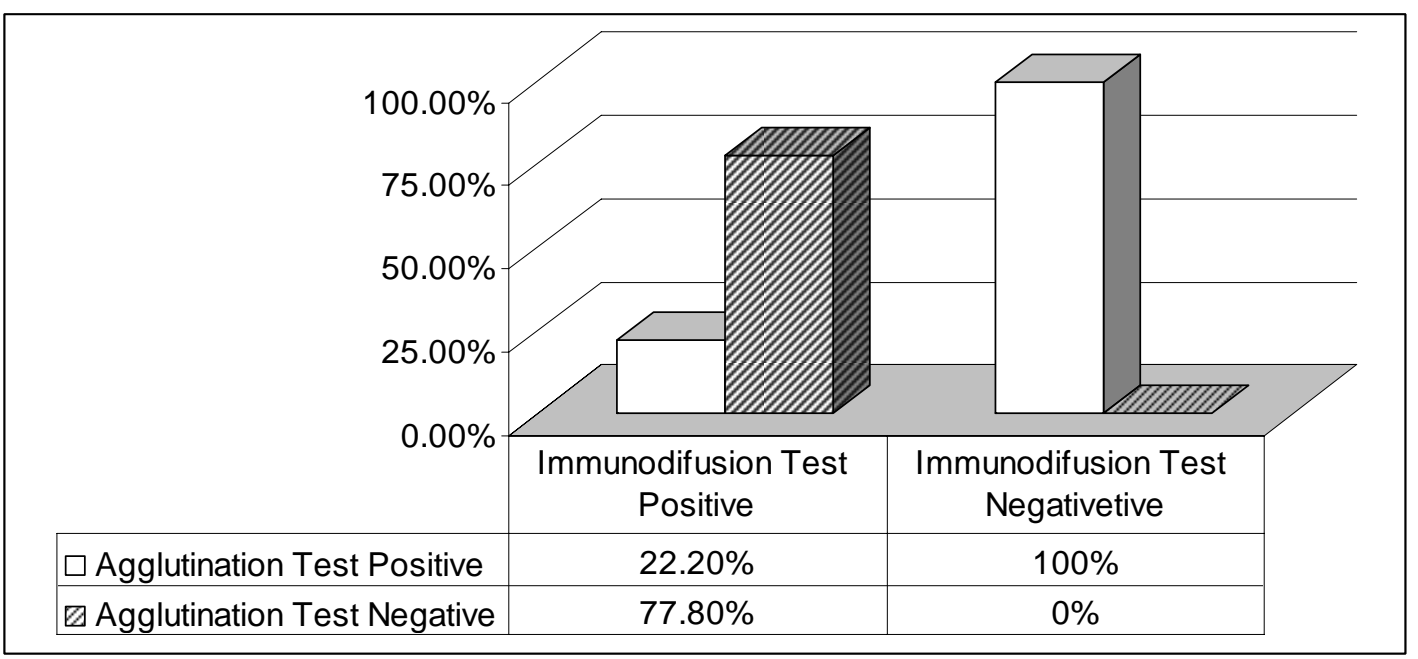

Figure-1: Comparison between the results of the immunodiffusion test and the agglutination test. 
Table-1 summarizes the agglutination test true positives and true negatives samples based on the results of immunodiffusion test. Taking into consideration the very high capability of immunodiffusion test to detect alloantibodies, the conditional ratios were calculated for agglutination test and are given underneath table 1. Agglutination test has a very high specifity and positive predictive value (PPV) (100\% for both). On the other hand, negative predictive value (NPV) reaches $87.9 \%$ and Sensitivity drops to $22.2 \%$. The accuracy of agglutination test to detect alloantibodies is found to be $88.3 \%$.

Table-1: Detection rate of alloantibody in patients with repeated blood transfusion

\begin{tabular}{ll|ccc}
\hline & & \multicolumn{3}{|c}{ Immunodiffusion Test } \\
& & Positive & Negative & Total \\
\hline \multirow{2}{*}{ Agglutination } & Positive & 2 & 0 & 2 \\
Test & Negative & 7 & 51 & 58 \\
& Total & 9 & 51 & 60 \\
\hline
\end{tabular}

Pearson Chi-Square value $=11.72$

$\mathrm{P}=0.001$

Sensitivity $=22.2 \%$

Specifity $=100 \%$

Positive predictive value $=100 \%$

Negative predictive value $=87.9 \%$

Accuracy $=88.3 \%$

\section{Discussion:}

Usually the tube technique has been employed to detect the presence of alloantibodies in particular those have the capacity to induce delayed haemolysis. Nevertheless a lot of experiments may be needed for detection of such antibodies. In an attempt to overcome this practical difficulty of the Tube technique, the DiaMed-ID® micro typing system (ID - gel test) was introduced by Lapierre $^{16}$ and is now commercially available in Sudan.

Patients of this study developed at least 2-3 episodes of delayed hemolysis occurring not less than 10 days after RBC transfusion. The tube agglutination test detected four alloantibodies and the immune-diffusion gel test detected nine allo-antibodies. Agglutination test achieved $100 \%$ specificity, however, this extremely high specificity score was at the expense of sensitivity, which achieved incredibly poor performance $(22.2 \%)$. The specificity of a test is the probability that a test will produce a true negative result when used in a patients with no alloantibodies (as determined by immunodiffusion test) while sensitivity is the probability that the test will produce a true positive result when used in patients with alloantibodies

Regarding predictive values, Agglutination test achieved $100 \%$ PPV, not far away NPV drops to $87.9 \%$. PPV refer to the probability that a person has alloantibodies when a positive test result is observed while NPV is the probability that a person has no alloantibodies when the test result is negative. Although, PPV is very good in this study, agglutination test failed to diagnose $77.8 \%$ of immunodiffusion test positive samples. This explains the relatively lower scores of NPV and sensitivity. However, it is worth mentioning that in practice, predictive values should only be calculated from cohort studies or studies that legitimately reflect the number of people in that population who have the disease of interest at that time. This is because predictive values are inherently dependent upon the prevalence of the disease.

Diagnostic likelihood ratios are not yet commonly reported in peer-reviewed literature, but they can be a valuable tool for comparing the accuracy of several tests to the gold standard, and they are not dependent 
upon the prevalence of disease. The positive diagnostic likelihood ratio (PDLR) of agglutination test $=22.2 / 0=\infty$ PDLR represents the odds ratio that a positive test result will be observed in population with alloantibodies compared to the odds that the same result will be observed among a population with no alloantibodies. In the current study, PDLR is very high indicating the usefulness of agglutination test. This is because the less useful tests will have smaller values. The negative diagnostic likelihood ratio $(\mathrm{PDLR})=7 / 51=0.13$. NDLR represents the odds ratio that a negative test result will be observed in patients with no alloantibodies compared to the odds that the same result will be observed among patients with no alloantibodies. Useful tests will, therefore, have NDLR close to zero making the current result moderately satisfactory.

\section{Conclusion}

The use of gel test has increased the number of antibodies detected. The accuracy (i.e. the proportion of both true positives and true negatives) of agglutination test to detect presence or absence of alloantibodies was found to be $88.3 \%$. Although there are no false positive samples, the false negative rate reached $77.8 \%$. Although agglutination test reduces transfusion reactions, transfusion of agglutination test negative blood can still induce post-transfusion reactions. Therefore, screening for unexpected antibodies using Immuno-Diffusion microtyping system should be part of all pre-transfusion testing.

\section{References:}

1. Landsteiner K, Wiener AS. An agglutinable factor in human blood recognized by immune sera for rhesus blood. Proc Soc Exp Biol Med. 1940;43:223.

2. Castro O, Sandler SG, Houston-Yu P et al. Predicting the effect of transfusing only phenotypematched RBCs to patients with sickle cell disease: theoretical and practical implications. Transfusion 2002;42:684-90.
3. Rosse WF, Gallagher D, Kinney TR et al. Cooperative Study of Sickle Cell Disease. Transfusion and alloimmunization in sickle cell disease. Blood 1990; 76: 1431-1437.

4. Davies SC \& Harewood MR. Blood transfusion in sickle cell disease. Blood Reviews 1997; 11: 57-71.

5. Vichinsky E, Earles A, Johnson $\mathrm{R}$ et al. Alloimmunization in sickle cell anemia and transfusion of racially unmatched blood. N Engl J Med.1990; 322 : $1617-1621$.

6. Aygun B, Padmanabham S, Paley C et al. Clinical significance of RBC alloantibodies and autoantibodies in sickle cell patients who received transfusions. Transfusion.2002; $42: 37-43$.

7. Syed SK, Sears DA, Werch JB et al. Case reports: delayed hemolytic transfusion reaction in sickle cell disease. Am J Med Sci 1996; 312:175-81.

8. Noizat-Pirenne F, Lee K, Pennec PY et al. Rare RHCE phenotypes in black individuals of AfroCaribbean origin: identification and transfusion safety. Blood 2002; 100:4223-31.

9. Talano JA, Hillery CA, Gottschall JL et al. Delayed hemolytic transfusion reaction/hyperhemolysis syndrome in children with sickle cell disease. Pediatrics 2003; 111:e661-5.

10. McGlennan AP, Grundy EM. Delayed haemolytic transfusion reaction and hyperhaemolysis complicating peri-operative blood transfusion in sickle cell disease. Anaesthesia 2005; 60:609-12.

11. Hannema SE, Brand A, van Meurs A et al. Delayed hemolytic transfusion reaction with hyperhemolysis after first red blood cell transfusion in child with betathalassemia: challenges in treatment. Transfusion. 2010 Feb;50(2):429-32. Epub 2009 Sep 24.

12. Chapman JF, Elliott C, Knowles SM et al. Guidelines for compatibility procedures in blood transfusion laboratories. Transfus Med. 2004 Feb;14(1):59-73.

13. Andrew H. Lichtman (2005) Boston, Massachusetts. Main edition ISBN: 1-4160-2389-5. International edition ISBN: 0-8089-2341-2.

14. Win N, Doughty H, Telfer P et al. Hyperhemolytic transfusion reaction in sickle cell disease. Transfusion.2001; $41: 323-328$.

15. Cox J, Steane E, Cunningham G et al. Risk of alloimmunization and delayed hemolytic transfusion reactions in patients with sickle cell disease. Arch Intern Med.1988; 148 :2485 -2489.

16. Lapierre, Y., Rigal, D., Adam, J et al. The Gel Test: a new way to detect red cell antigen - antibody reactions. Transfusion 1990; 30: $109-113$. 